RAD HAZU. MATEMATIČKE ZNANOSTI

Vol. $23=538(2019): 85-105$

DOI: https://doi.org/10.21857/mnlqgc00ny

\title{
RESOLVENT OPERATOR OF SINGULAR DIRAC SYSTEM WITH TRANSMISSION CONDITIONS
}

\author{
Bilender P. Allahverdiev and Hüseyin Tuna
}

\begin{abstract}
This paper is concerned with the resolvent operator of one dimensional singular Dirac operator with transmission conditions. We study the Titchmarsh-Weyl function of this problem. Later, we construct a Green function and a spectral function for regular and singular problems. With the help of these functions, we obtain an expansion into a Fourier series of resolvent in regular case. Furthermore, we give integral representations in terms of the spectral function for the resolvent of this operator with transmission conditions in singular case. Finally, we obtain a formula for the Titchmarsh-Weyl function in terms of the spectral function of the singular Dirac system.
\end{abstract}

\section{INTRODUCTION}

Recently, much attention has been paid to the boundary value problems with transmission conditions, or discontinuous boundary value problems. It has been shown that they are closely related to various physically interesting models and theories, such as the heat and mass transfer theory (see [22]), radio science (see [23]), and geophysics (see [19]). For further references consider [1-16, 19, 21-28, 30, 32, 34-37].

On the other hand, the Dirac systems play an important role in the theory of relativistic quantum mechanics since basic physics of relativistic quantum mechanics was governed by the Dirac operators. For example, they predict the existence of a positron and elucidate the origin of spin $1 / 2$ of an electron (see [29]). Dirac systems in the finite interval have been considered in [20], [33] whereas the Dirac system in the infinite interval were considered in [20]. Direct or inverse spectral problems for Dirac operators with transmission conditions were studied in $[6-8,13,16,24,36]$. In [14], Hira and Altınışı investigated asymptotic behavior of eigenvalues and eigenfunctions of discontinuous Dirac system which includes an eigenvalue parameter in a

2010 Mathematics Subject Classification. 34L40, 34A36, 34A37, 47E05, 34B20, 34L10.

Key words and phrases. Dirac operator, transmission conditions, singular point, spectral function, Titchmarsh-Weyl function, resolvent operator. 
transmission condition. In [30], Tharwat and Bhrawy computed the eigenvalues of a discontinuous regular Dirac systems with transmission conditions at the point of discontinuity numerically. In [15], Kablan and Özden studied a Dirac system with transmission conditions and eigenparameter in the boundary condition. They investigated the existence of the solution and some spectral properties of this problem. For the classical Sturm-Liouville equation, the integral representation of the resolvent was first proved by H. Weyl in 1910. Similar theorems were proved by E. C. Titchmarsh [31] and [20]. Levitan and Sargsjan obtained the integral representation of the resolvent for one dimensional Dirac operators ([20]).

The main result of the paper is obtaining an integral representations in terms of the spectral function for the resolvent of the one dimensional singular Dirac operator with transmission conditions.

This paper is organized as follows. In Section 2, we consider one dimensional singular Dirac operator with transmission (or impulsive) conditions. In Section 3, we study the Titchmarsh-Weyl function of one dimensional singular Dirac operator with transmission conditions. We will define limit-point and limit-circle singularities. In Section 4, we construct a Green function and a spectral function for regular problem. With the help of these functions, we obtain an expansion into a Fourier series of resolvent in regular case. In Section 5, we give the main result of this paper. We obtain integral representations in terms of the spectral function for the resolvent of this operator with transmission conditions in singular case. Finally, in Section 6, we obtain a formula for the Titchmarsh-Weyl function in terms of the spectral function of the singular Dirac system.

\section{Preliminaries}

We consider one dimensional Dirac expression

$$
\tau(y):=\left(\begin{array}{cc}
0 & -1 \\
1 & 0
\end{array}\right) \frac{d y(x)}{d x}+Q(x) y(x), x \in \Omega:=\Omega_{1} \cup \Omega_{2},
$$

where

$$
y(x)=\left(\begin{array}{l}
y_{1}(x) \\
y_{2}(x)
\end{array}\right), Q(x)=\left(\begin{array}{cc}
p(x) & 0 \\
0 & r(x)
\end{array}\right),
$$

and $\Omega_{1}:=[a, c), \Omega_{2}:=(c, b],-\infty<a<c<b<+\infty$. We assume that the points $a, b$ and $c$ are regular for the differential expression $\tau$. $p$ and $r$ are realvalued, Lebesgue measurable functions on $\Omega$ and $p, r \in L^{1}\left(\Omega_{k}\right),(k=1,2)$. The point $c$ is regular if $p, r \in L^{1}[c-\epsilon, c+\epsilon]$ for some $\epsilon>0$.

Let us consider the Dirac system

$$
\tau(y)=\lambda y, x \in \Omega,
$$


with the boundary conditions

$$
\begin{aligned}
& y_{1}(a) \cos \beta+y_{2}(a) \sin \beta=0, \\
& y_{1}(b) \cos \alpha+y_{2}(b) \sin \alpha=0, \alpha, \beta \in \mathbb{R}:=(-\infty, \infty),
\end{aligned}
$$

and transmission (or impulsive) conditions

$$
y(c+)=C y(c-), C \in M_{2}(\mathbb{R}), \operatorname{det} C=\gamma>0,
$$

where $\lambda$ is a complex spectral parameter and $M_{2}(\mathbb{R})$ denotes the the $2 \times 2$ matrices with entries from $\mathbb{R}$.

Now, we introduce the direct sum Hilbert space $H=L^{2}\left(\Omega_{1} ; E\right) \dot{+}$ $L^{2}\left(\Omega_{2} ; E\right)$ (where $E:=\mathbb{C}^{2}$ ) of vector-valued functions with values in $\mathbb{C}^{2}$ and with the inner product

$$
\langle u, v\rangle_{H}:=\int_{a}^{c}(u(x), v(x))_{E} d x+\gamma \int_{c}^{b}(u(x), v(x))_{E} d x, \delta=\frac{1}{\gamma}
$$

where

$$
\begin{gathered}
u(x)=\left(\begin{array}{l}
u_{1}(x) \\
u_{2}(x)
\end{array}\right), v(x)=\left(\begin{array}{l}
v_{1}(x) \\
v_{2}(x)
\end{array}\right), \\
u_{1}(x)=\left\{\begin{array}{ll}
u_{1}^{(1)}(x), & x \in \Omega_{1} \\
u_{1}^{(2)}(x), & x \in \Omega_{2}
\end{array}, \quad u_{2}(x)=\left\{\begin{array}{ll}
u_{2}^{(1)}(x), & x \in \Omega_{1} \\
u_{2}^{(2)}(x), & x \in \Omega_{2}
\end{array},\right.\right. \\
v_{1}(x)=\left\{\begin{array}{ll}
v_{1}^{(1)}(x), & x \in \Omega_{1} \\
v_{1}^{(2)}(x), & x \in \Omega_{2}
\end{array}, v_{2}(x)= \begin{cases}v_{2}^{(1)}(x), & x \in \Omega_{1} \\
v_{2}^{(2)}(x), & x \in \Omega_{2}\end{cases} \right.
\end{gathered}
$$

Denote by $D$ the linear set of all vector-valued functions $y \in H$ such that $y_{1}, y_{2}$ are locally absolutely continuous functions on $\Omega$, one-sided limits $y_{1}(c \pm), y_{2}(c \pm)$ exist and are finite and $\tau(y) \in H$. The operator $L$ defined by $L y=\tau(y)(y \in D)$ is called the maximal operator on $H$.

For two arbitrary vector-valued functions $y, z \in D$, we have Green's formula

$$
\int_{a}^{b} \tau(y) \bar{z} d x-\int_{a}^{b} y \overline{\tau(z)} d x=[y, z]_{c-}-[y, z]_{a}+[y, z]_{b}-[y, z]_{c+}
$$

where $[y, z]_{x}:=W_{x}(y, \bar{z}):=y_{1}(x) \overline{z_{2}(x)}-y_{2}(x) \overline{z_{1}(x)}(x \in \Omega)$.

Now, we introduce the Hilbert space $\mathcal{H}:=L^{2}\left(\Omega_{1}\right)+L^{2}\left(\Omega_{3}\right),\left(\Omega_{1}:=\right.$ $\left.[a, c), \Omega_{3}:=(c, \infty)\right)$ with the inner product

$$
\langle u, v\rangle_{\mathcal{H}}:=\int_{a}^{c}(u(x), v(x))_{E} d x+\delta \int_{c}^{\infty}(u(x), v(x))_{E} d x, \delta=\frac{1}{\gamma}
$$


where

$$
\begin{aligned}
& u(x)=\left(\begin{array}{l}
u_{1}(x) \\
u_{2}(x)
\end{array}\right), v(x)=\left(\begin{array}{l}
v_{1}(x) \\
v_{2}(x)
\end{array}\right), \\
& u_{1}(x)=\left\{\begin{array}{ll}
u_{1}^{(1)}(x), & x \in \Omega_{1} \\
u_{1}^{(2)}(x), & x \in \Omega_{3}
\end{array}, u_{2}(x)=\left\{\begin{array}{ll}
u_{2}^{(1)}(x), & x \in \Omega_{1} \\
u_{2}^{(2)}(x), & x \in \Omega_{3}
\end{array},\right.\right. \\
& v_{1}(x)=\left\{\begin{array}{ll}
v_{1}^{(1)}(x), & x \in \Omega_{1} \\
v_{1}^{(2)}(x), & x \in \Omega_{3}
\end{array}, v_{2}(x)=\left\{\begin{array}{cc}
v_{2}^{(1)}(x), & x \in \Omega_{1} \\
v_{2}^{(2)}(x), & x \in \Omega_{3}
\end{array} .\right.\right.
\end{aligned}
$$

We will denote by

$$
\begin{gathered}
\varphi(x, \lambda)=\left(\begin{array}{l}
\varphi_{1}(x, \lambda) \\
\varphi_{2}(x, \lambda)
\end{array}\right), \varphi_{1}(x, \lambda)= \begin{cases}\phi_{1}^{(1)}(x, \lambda), & x \in \Omega_{1} \\
\phi_{1}^{(2)}(x, \lambda), & x \in \Omega_{3}\end{cases} \\
\varphi_{2}(x, \lambda)= \begin{cases}\varphi_{2}^{(1)}(x, \lambda), & x \in \Omega_{1} \\
\varphi_{2}^{(2)}(x, \lambda), & x \in \Omega_{3}\end{cases}
\end{gathered}
$$

and

$$
\begin{gathered}
\theta(x, \lambda)=\left(\begin{array}{c}
\theta_{1}(x, \lambda) \\
\theta_{2}(x, \lambda)
\end{array}\right), \theta_{1}(x, \lambda)=\left\{\begin{array}{ll}
\theta_{1}^{(1)}(x, \lambda), & x \in \Omega_{1} \\
\theta_{1}^{(2)}(x, \lambda), & x \in \Omega_{3}
\end{array},\right. \\
\theta_{2}(x, \lambda)= \begin{cases}\theta_{2}^{(1)}(x, \lambda), & x \in \Omega_{1} \\
\theta_{2}^{(2)}(x, \lambda), & x \in \Omega_{3}\end{cases}
\end{gathered}
$$

the solution of the system $\tau(y)=\lambda y, x \in \Omega_{1} \cup \Omega_{3}$ which satisfy the initial conditions

$$
\begin{gathered}
\varphi_{1}^{(1)}(a, \lambda)=\sin \beta, \varphi_{2}^{(1)}(a, \lambda)=-\cos \beta, \\
\theta_{1}^{(1)}(a, \lambda)=\cos \beta, \theta_{2}^{(1)}(a, \lambda)=\sin \beta .
\end{gathered}
$$

and transmission conditions

$$
\begin{gathered}
\Phi(c+, \lambda)=C \Phi(c-, \lambda), \Phi(x, \lambda):=\left(\begin{array}{c}
\varphi_{1}(x, \lambda) \\
\varphi_{2}(x, \lambda)
\end{array}\right) \\
\Theta(c+, \lambda)=C \Theta(c-, \lambda), \Theta(x, \lambda):=\left(\begin{array}{c}
\theta_{1}(x, \lambda) \\
\theta_{2}(x, \lambda)
\end{array}\right), \\
C \in M_{2}(\mathbb{R}), \operatorname{det} C=\gamma>0 .
\end{gathered}
$$

\section{The Titchmarsh-Weyl Function}

In this section, we will consider the Titchmarsh-Weyl function of one dimensional singular Dirac operator with transmission conditions. Later, we will define limit-point and limit-circle singularities. 
We will denote by $\theta(x, \lambda)+m_{b}(\lambda) \varphi(x, \lambda)$ the solution of the equation (2.1) which satisfy the boundary condition

$$
\begin{aligned}
& \left(\theta_{1}^{(2)}(b, \lambda)+m_{b}(\lambda) \varphi_{1}^{(2)}(b, \lambda)\right) \cos \alpha \\
& +\left(\theta_{2}^{(2)}(b, \lambda)+m_{b}(\lambda) \varphi_{2}^{(2)}(b, \lambda)\right) \sin \alpha=0 .
\end{aligned}
$$

Then, $m_{b}(\lambda)$ satisfies the relation

$$
m_{b}(\lambda)=-\frac{\theta_{1}^{(2)}(b, \lambda) \cot \alpha+\theta_{2}^{(2)}(b, \lambda)}{\varphi_{1}^{(2)}(b, \lambda) \cot \alpha+\varphi_{2}^{(2)}(b, \lambda)} .
$$

It is clear that $m_{b}(\lambda)$ is a meromorphic function of $\lambda$, since $\theta(x, \lambda)$ and $\varphi(x, \lambda)$ are entire functions of $\lambda$. Furthermore, since the eigenvalues of the regular problem are real, all poles of $m_{b}(\lambda)$ are real and simple. The function $m_{b}$ is called the Titchmarsh-Weyl function of the regular problem (1)-(4). If $\cot \beta$ is replaced by a complex variable $z$, then we have

$$
m_{b}(\lambda, z)=-\frac{\theta_{1}^{(2)}(b, \lambda) z+\theta_{2}^{(2)}(b, \lambda)}{\varphi_{1}^{(2)}(b, \lambda) z+\varphi_{2}^{(2)}(b, \lambda)} .
$$

For every $\lambda$, the equality (3.1) is a one-to-one conformal mapping in $z$, which follows from the theory of Möbius transformations [17]. Hence, if $\operatorname{Im} \lambda \neq 0$, then $m_{b}(\lambda, z)$ varies on a circle $C_{b}(\lambda)$ with a finite radius in the $m_{b}$-plane as $z$ varies over the real axis of the $z$-plane.

Using this notation we now state the result from [6].

TheOREm 3.1. Let $\theta(x, \lambda), \varphi(x, \lambda)$ be two linearly independent solution of the system (2.1) satisfying the initial conditions (2.7) and transmission conditions (2.8). Then, the solution

$$
\omega(x, \lambda)=\theta(x, \lambda)+m_{b}(\lambda) \varphi(x, \lambda)
$$

satisfies the boundary condition

$$
\begin{gathered}
\left(\theta_{1}^{(2)}(b, \lambda)+m_{b}(\lambda) \varphi_{1}^{(2)}(b, \lambda)\right) \cos \alpha \\
+\left(\theta_{2}^{(2)}(b, \lambda)+m_{b}(\lambda) \varphi_{2}^{(2)}(b, \lambda)\right) \sin \alpha=0 .
\end{gathered}
$$

if and only if $m_{b}(\lambda)$ is on $C_{b}$ with

$$
\lim _{b \rightarrow \infty} W(\omega, \bar{\omega})(b, \lambda)=0 .
$$

If $b \rightarrow \infty$, then $C_{b}$ tends either to limit-circle $C_{\infty}$ or to the limit-point $m_{\infty}$. In the first case, all solutions of the system are in the space $\mathcal{H}$. In the second case, if $\operatorname{Im} \lambda \neq 0$, one linearly independent solution is in the space $\mathcal{H}$. In the limit-circle case, a point is on $C_{\infty}$ if and only if

$$
\lim _{b \rightarrow \infty} W(\omega, \bar{\omega})(b, \lambda)=0
$$


The function $m(\lambda):=\lim _{b \rightarrow \infty} m_{b}(\lambda)$ is called the Titchmars-Weyl function, and $\chi(x, \lambda):=\theta(x, \lambda)+m(\lambda) \varphi(x, \lambda)$ is called the Weyl solution of the singular system $\tau(y)=\lambda y\left(x \in \Omega_{1} \cup \Omega_{3}\right)$ satisfying (2.2), (2.4).

Let us define

$$
\chi_{b}(x, \lambda):=\theta(x, \lambda)+m_{b}(\lambda) \varphi(x, \lambda), x \in \Omega .
$$

Then, we have the following lemma.

Lemma 3.2. For each nonreal $\lambda$, we have

$$
\begin{gathered}
\chi_{b}(x, \lambda) \rightarrow \chi(x, \lambda), b \rightarrow \infty \\
\int_{a}^{c}\left\|\chi_{b}^{(1)}(x, \lambda)\right\|_{E}^{2} d x+\delta \int_{c}^{b}\left\|\chi_{b}^{(2)}(x, \lambda)\right\|_{E}^{2} d x \\
\rightarrow \int_{a}^{c}\left\|\chi^{(1)}(x, \lambda)\right\|_{E}^{2} d x+\delta \int_{c}^{\infty}\left\|\chi^{(2)}(x, \lambda)\right\|_{E}^{2} d x, b \rightarrow \infty .
\end{gathered}
$$

Proof. It is clear that

$$
\chi_{b}(x, \lambda)=\chi(x, \lambda)+\left\{m_{b}(\lambda)-m(\lambda)\right\} \varphi(x, \lambda),
$$

where $\chi(x, \lambda) \in \mathcal{H}$ and $m_{b}(\lambda)$ is a point of the circle. According to [20], [6]

$$
\begin{aligned}
\left|m_{b}(\lambda)-m(\lambda)\right| & \leq 2 r_{b}(\lambda) \\
& =\left[|v|\left(\begin{array}{c}
\int_{a}^{c}\left\|\varphi^{(1)}(x, \lambda)\right\|_{E}^{2} d x \\
+\delta \int_{c}^{b}\left\|\varphi^{(2)}(x, \lambda)\right\|_{E}^{2} d x
\end{array}\right)\right]^{-1}, \operatorname{Im} \lambda=v \neq 0 .
\end{aligned}
$$

Since $r_{b}(\lambda) \rightarrow 0$, we get $\chi_{b}(x, \lambda) \rightarrow \chi(x, \lambda)(b \rightarrow \infty)$. Furthermore, we have

$$
\begin{aligned}
& \int_{a}^{c}\left\|\left\{m_{b}(\lambda)-m(\lambda)\right\} \varphi^{(1)}(x, \lambda)\right\|_{E}^{2} d x \\
& \quad+\delta \int_{c}^{b}\left\|\left\{m_{b}(\lambda)-m(\lambda)\right\} \varphi^{(2)}(x, \lambda)\right\|_{E}^{2} d x \\
& =\left|m_{b}(\lambda)-m(\lambda)\right|^{2} \int_{a}^{c}\left\|\varphi^{(1)}(x, \lambda)\right\|_{E}^{2} d x \\
& \quad+\delta\left|m_{b}(\lambda)-m(\lambda)\right|^{2} \int_{c}^{b}\left\|\varphi^{(2)}(x, \lambda)\right\|_{E}^{2} d x \\
& \leq\left(|v|^{2}\left[\int_{a}^{c}\left\|\varphi^{(1)}(x, \lambda)\right\|_{E}^{2} d x+\delta \int_{c}^{b}\left\|\varphi^{(2)}(x, \lambda)\right\|_{E}^{2} d x\right]\right)^{-1} .
\end{aligned}
$$

Therefore, we get

$$
\begin{aligned}
& \int_{a}^{c}\left\|\chi_{b}^{(1)}(x, \lambda)\right\|_{E}^{2} d x+\delta \int_{c}^{b}\left\|\chi_{b}^{(2)}(x, \lambda)\right\|_{E}^{2} d x \\
& \rightarrow \int_{a}^{c}\left\|\chi^{(1)}(x, \lambda)\right\|_{E}^{2} d x+\delta \int_{c}^{\infty}\left\|\chi^{(2)}(x, \lambda)\right\|_{E}^{2} d x, b \rightarrow \infty .
\end{aligned}
$$




\section{An EXPANSion of the RESOlVENT IN REGUlar CASE}

In this section, we will obtain an expansion into a Fourier series of resolvent in regular case. We will construct a Green function and a spectral function for this problem. With the help of these functions, we will obtain an expansion into a Fourier series of resolvent.

Let two functions $\chi_{b}(x, \lambda)$ and $\varphi(x, \lambda)$ be as in $(3.2)$ and $(2.6)$, respectively. Putting

$$
= \begin{cases}\left(\begin{array}{ll}
\chi_{b 1}(x, \lambda) \varphi_{1}(t, \lambda) & \chi_{b 1}(x, \lambda) \varphi_{2}(t, \lambda) \\
\chi_{b 2}(x, \lambda) \varphi_{1}(t, \lambda) & \chi_{b 2}(x, \lambda) \varphi_{2}(t, \lambda)
\end{array}\right), \quad t \leq x, x \neq c, t \neq c \\
\left(\begin{array}{ll}
\varphi_{1}(x, \lambda) \chi_{b 1}(t, \lambda) & \varphi_{1}(x, \lambda) \chi_{b 2}(t, \lambda) \\
\varphi_{2}(x, \lambda) \chi_{b 1}(t, \lambda) & \varphi_{2}(x, \lambda) \chi_{b 2}(t, \lambda)
\end{array}\right), \quad x<t, x \neq c, t \neq c,\end{cases}
$$

$$
\left(R_{b} f\right)(x, \lambda)=y(x, \lambda)=\int_{a}^{b} G_{b}(x, t, \lambda) f(t) d t, \lambda \in \mathbb{C} .
$$

Hence, we have

$$
\begin{aligned}
& G_{b}(x, t, \lambda) f(t) \\
& =\left\{\begin{array}{c}
\chi_{b 1}(x, \lambda) \varphi_{1}(t, \lambda) f_{1}(t) \\
+\chi_{b 1}(x, \lambda) \varphi_{2}(t, \lambda) f_{2}(t) \\
\chi_{b 2}(x, \lambda) \varphi_{1}(t, \lambda) f_{1}(t) \\
+\chi_{b 2}(x, \lambda) \varphi_{2}(t, \lambda) f_{2}(t)
\end{array}\right), \quad t \leq x, x \neq c, t \neq c \\
& \left(\begin{array}{c}
\varphi_{1}(x, \lambda) \chi_{b 1}(t, \lambda) f_{1}(t) \\
+\varphi_{1}(x, \lambda) \chi_{b 2}(t, \lambda) f_{2}(t) \\
\varphi_{2}(x, \lambda) \chi_{b 1}(t, \lambda) f_{1}(t) \\
+\varphi_{2}(x, \lambda) \chi_{b 2}(t, \lambda) f_{2}(t)
\end{array}\right), \quad x<t, x \neq c, t \neq c .
\end{aligned}
$$

Now, we shall show that (4.2) satisfies the equation $\tau(y)=\lambda y+f(x \in \Omega$, $f \in H$ ) and the conditions (2.2)-(2.4). From (4.2), we have

$$
y_{1}(x, \lambda)=\left\{\begin{array}{c}
\chi_{b 1}^{(1)}(x, \lambda) \int_{a}^{x}\left(\varphi_{1}^{(1)}(t, \lambda) f_{1}(t)+\varphi_{2}^{(1)}(t, \lambda) f_{2}(t)\right) d t \\
+\varphi_{1}^{(1)}(x, \lambda) \int_{x}^{c}\left(\chi_{b 1}^{(1)}(t, \lambda) f_{1}(t)+\chi_{b 2}^{(1)}(t, \lambda) f_{2}(t)\right) d t \\
+\varphi_{1}^{(1)}(x, \lambda) \delta \int_{c}^{b}\left(\chi_{b 1}^{(2)}(t, \lambda) f_{1}(t)+\chi_{b 2}^{(2)}(t, \lambda) f_{2}(t)\right) d t, x \in \Omega_{1}, \\
\chi_{b 1}^{(2)}(x, \lambda) \int_{a}^{c}\left(\varphi_{1}^{(1)}(t, \lambda) f_{1}(t)+\varphi_{2}^{(1)}(t, \lambda) f_{2}(t)\right) d t \\
+\chi_{b 1}^{(2)}(x, \lambda) \delta \int_{c}^{x}\left(\varphi_{1}^{(2)}(t, \lambda) f_{1}(t)+\varphi_{2}^{(2)}(t, \lambda) f_{2}(t)\right) d t \\
+\varphi_{1}^{(2)}(x, \lambda) \delta \int_{x}^{b}\left(\chi_{b 1}^{(2)}(t, \lambda) f_{1}(t)+\chi_{b 2}^{(2)}(t, \lambda) f_{2}(t)\right) d t, x \in \Omega_{2}
\end{array}\right.
$$


and

$$
y_{2}(x, \lambda)=\left\{\begin{array}{c}
\chi_{b 2}^{(1)}(x, \lambda) \int_{a}^{x}\left(\varphi_{1}^{(1)}(t, \lambda) f_{1}(t)+\varphi_{2}^{(1)}(t, \lambda) f_{2}(t)\right) d t \\
+\varphi_{2}^{(1)}(x, \lambda) \int_{x}^{c}\left(\chi_{b 1}^{(1)}(t, \lambda) f_{1}(t)+\chi_{b 2}^{(1)}(t, \lambda) f_{2}(t)\right) d t \\
+\varphi_{2}^{(1)}(x, \lambda) \delta \int_{c}^{b}\left(\chi_{b 1}^{(2)}(t, \lambda) f_{1}(t)+\chi_{b 2}^{(2)}(t, \lambda) f_{2}(t)\right) d t, x \in \Omega_{1}, \\
\chi_{b 2}^{(2)}(x, \lambda) \int_{a}^{c}\left(\varphi_{1}^{(1)}(t, \lambda) f_{1}(t)+\varphi_{2}^{(1)}(t, \lambda) f_{2}(t)\right) d t \\
+\chi_{b 2}^{(2)}(x, \lambda) \delta \int_{c}^{x}\left(\varphi_{1}^{(2)}(t, \lambda) f_{1}(t)+\varphi_{2}^{(2)}(t, \lambda) f_{2}(t)\right) d t \\
+\varphi_{2}^{(2)}(x, \lambda) \delta \int_{x}^{b}\left(\chi_{b 1}^{(2)}(t, \lambda) f_{1}(t)+\chi_{b 2}^{(2)}(t, \lambda) f_{2}(t)\right) d t, x \in \Omega_{2} .
\end{array}\right.
$$

From (4.3), it follows that

$$
y_{1}^{\prime}(x, \lambda)=\left\{\begin{array}{c}
\chi_{b 1}^{(1) \prime}(x, \lambda) \int_{a}^{x}\left(\varphi_{1}^{(1)}(t, \lambda) f_{1}(t)+\varphi_{2}^{(1)}(t, \lambda) f_{2}(t)\right) d t \\
+\varphi_{1}^{(1) \prime}(x, \lambda) \int_{x}^{c}\left(\chi_{b 1}^{(1)}(t, \lambda) f_{1}(t)+\chi_{b 2}^{(1)}(t, \lambda) f_{2}(t)\right) d t \\
+\varphi_{1}^{(1) \prime}(x, \lambda) \delta \int_{c}^{b}\left(\chi_{b 1}^{(2)}(t, \lambda) f_{1}(t)+\chi_{b 2}^{(2)}(t, \lambda) f_{2}(t)\right) d t \\
+W\left(\varphi, \chi_{b}\right) f_{2}(x), x \in \Omega_{1}, \\
\chi_{b 1}^{(2) \prime}(x, \lambda) \int_{a}^{c}\left(\varphi_{1}^{(1)}(t, \lambda) f_{1}(t)+\varphi_{2}^{(1)}(t, \lambda) f_{2}(t)\right) d t \\
+\chi_{b 1}^{(2) \prime}(x, \lambda) \delta \int_{c}^{x}\left(\varphi_{1}^{(2)}(t, \lambda) f_{1}(t)+\varphi_{2}^{(2)}(t, \lambda) f_{2}(t)\right) d t \\
+\varphi_{1}^{(2) \prime}(x, \lambda) \delta \int_{x}^{b}\left(\chi_{b 1}^{(2)}(t, \lambda) f_{1}(t)+\chi_{b 2}^{(2)}(t, \lambda) f_{2}(t)\right) d t \\
+W\left(\varphi, \chi_{b}\right) f_{2}(x), x \in \Omega_{2}
\end{array}\right.
$$

$$
=\left\{\begin{array}{c}
\{\lambda-r(x)\} \chi_{b 2}^{(1)}(x, \lambda) \int_{a}^{x}\left(\varphi_{1}^{(1)}(t, \lambda) f_{1}(t)+\varphi_{2}^{(1)}(t, \lambda) f_{2}(t)\right) d t \\
\{\lambda-r(x)\} \varphi_{2}^{(1)}(x, \lambda)+\int_{x}^{c}\left(\chi_{b 1}^{(1)}(t, \lambda) f_{1}(t)+\chi_{b 2}^{(1)}(t, \lambda) f_{2}(t)\right) d t \\
\{\lambda-r(x)\} \varphi_{2}^{(1)}(x, \lambda) \delta \int_{c}^{b}\left(\chi_{b 1}^{(2)}(t, \lambda) f_{1}(t)+\chi_{b 2}^{(2)}(t, \lambda) f_{2}(t)\right) d t \\
\quad+f_{2}(x), x \in \Omega_{1}, \\
\{\lambda-r(x)\} \chi_{b 2}^{(2)}(x, \lambda) \int_{a}^{c}\left(\varphi_{1}^{(1)}(t, \lambda) f_{1}(t)+\varphi_{2}^{(1)}(t, \lambda) f_{2}(t)\right) d t \\
\{\lambda-r(x)\} \varphi_{2}^{(2)}(x, \lambda) \delta \int_{c}^{x}\left(\chi_{b 1}^{(2)}(t, \lambda) f_{1}(t)+\chi_{b 2}^{(2)}(t, \lambda) f_{2}(t)\right) d t \\
\{\lambda-r(x)\} \varphi_{2}^{(2)}(x, \lambda) \delta \int_{x}^{b}\left(\chi_{b 1}^{(2)}(t, \lambda) f_{1}(t)+\chi_{b 2}^{(2)}(t, \lambda) f_{2}(t)\right) d t \\
\quad+f_{2}(x), x \in \Omega_{2},
\end{array}\right.
$$$$
=\{\lambda-r(x)\} y_{2}(x, \lambda)+f_{2}(x) \text {. }
$$ 
The validity of of the other equation in (2.1) is proved similarly. Hence the function $y(x, \lambda)=\left(\begin{array}{l}y_{1}(x, \lambda) \\ y_{2}(x, \lambda)\end{array}\right)$ in (4.2) is the solution of the system $(2.1)$. We check at once that (4.2) satisfies the conditions (2.2)-(2.4).

Let $\lambda_{m, b}(m \in \mathbb{Z}:=\{0, \pm 1, \pm 2, \ldots\})$ denote the eigenvalues of the selfadjoint problem (1)-(4) and by

$$
\begin{aligned}
\phi_{m, b}(x) & =\left(\begin{array}{l}
\phi_{m, b 1}(x) \\
\phi_{m, b 2}(x)
\end{array}\right), \phi_{m, b 1}(x)=\left\{\begin{array}{ll}
\phi_{m, b 1}^{(1)}(x), & x \in \Omega_{1} \\
\phi_{m, b 2}^{(2)}(x), & x \in \Omega_{2}
\end{array},\right. \\
\phi_{m, b 2}(x) & = \begin{cases}\phi_{m, b 2}^{(1)}(x), & x \in \Omega_{1} \\
\phi_{m, b 2}^{(2)}(x), & x \in \Omega_{2}\end{cases}
\end{aligned}
$$

the corresponding eigenfunction which satisfy the conditions (2)-(4). If

$$
\begin{aligned}
f(x) & =\left(\begin{array}{l}
f_{1}(x) \\
f_{2}(x)
\end{array}\right), f_{1}(x)=\left\{\begin{array}{ll}
f_{1}^{(1)}(x), & x \in \Omega_{1} \\
f_{1}^{(2)}(x), & x \in \Omega_{2}
\end{array},\right. \\
f_{2}(x) & =\left\{\begin{array}{ll}
f_{2}^{(1)}(x), & x \in \Omega_{1} \\
f_{2}^{(2)}(x), & x \in \Omega_{2}
\end{array},\right.
\end{aligned}
$$

$f \in H$, and

$$
\begin{aligned}
\alpha_{m, b}^{2}= & \int_{a}^{c}\left(\left(\phi_{m, b 1}^{(1)}(x)\right)^{2}+\left(\phi_{m, b 2}^{(1)}(x)\right)^{2}\right) d x \\
& +\delta \int_{c}^{b}\left(\left(\phi_{m, b 1}^{(2)}(x)\right)^{2}+\left(\phi_{m, b 2}^{(2)}(x)\right)^{2}\right) d x
\end{aligned}
$$

Then we have

$$
\begin{aligned}
& \|f\|_{H}^{2}=\int_{a}^{c}\left(\left|f_{1}^{(1)}(x)\right|^{2}+\left|f_{2}^{(1)}(x)\right|^{2}\right) d x \\
& +\delta \int_{c}^{b}\left(\left|f_{1}^{(2)}(x)\right|^{2}+\left|f_{2}^{(2)}(x)\right|^{2}\right) d x \\
& (4.5)=\sum_{m=-\infty}^{\infty} \frac{1}{\alpha_{m, b}^{2}}\left\{\begin{array}{c}
\int_{a}^{c}\left(f_{1}^{(1)}(x) \phi_{m, b 1}^{(1)}(x)+f_{2}^{(1)}(x) \phi_{m, b 2}^{(1)}(x)\right) d x \\
+\delta \int_{c}^{b}\left(f_{1}^{(2)}(x) \phi_{m, b 1}^{(2)}(x)+f_{2}^{(2)}(x) \phi_{m, b 2}^{(2)}(x)\right) d x
\end{array}\right\}^{2}
\end{aligned}
$$

which is called the Parseval equality.

Now, let us define the nondecreasing step function $\varrho_{b}$ on $\mathbb{R}$ by

$$
\varrho_{b}(\lambda)=\left\{\begin{array}{cc}
-\sum_{\lambda<\lambda_{m, b}<0} \frac{1}{\alpha_{m, b}^{2}}, & \text { for } \lambda \leq 0 \\
\sum_{0 \leq \lambda_{m, b}<\lambda} \frac{1}{\alpha_{m, b}^{2}}, & \text { for } \lambda \geq 0 .
\end{array}\right.
$$


Then equalities (4.5) can be written as

$$
\|f\|_{H}^{2}=\int_{-\infty}^{\infty}|F(\lambda)|^{2} d \varrho_{b}(\lambda)
$$

where

$$
\begin{aligned}
F(\lambda)= & \int_{a}^{c}\left(f_{1}^{(1)}(x) \phi_{m, b 1}^{(1)}(x)+f_{2}^{(1)}(x) \phi_{m, b 2}^{(1)}(x)\right) d x \\
& +\delta \int_{c}^{b}\left(f_{1}^{(2)}(x) \phi_{m, b 1}^{(2)}(x)+f_{2}^{(2)}(x) \phi_{m, b 2}^{(2)}(x)\right) d x .
\end{aligned}
$$

By letting $b \rightarrow \infty$, we have the Parseval equality for the problem (2.1), (2.2), (2.4).

Now, we will give a definition.

A function $f$ defined on an interval $[a, b]$ is said to be of bounded variation if there is a constant $C>0$ such that

$$
\sum_{k=1}^{n}\left|f\left(x_{k}\right)-f\left(x_{k-1}\right)\right| \leq C
$$

for every partition

$$
a=x_{0}<x_{1}<\ldots<x_{n}=b
$$

of $[a, b]$ by points of subdivision $x_{0}, x_{1}, \ldots, x_{n}$.

Let $f$ be a function of bounded variation. Then, by the total variation of $f$ on $[a, b]$, denoted by $\bigvee_{a}^{b}(f)$, we mean the quantity

$$
\bigvee_{a}^{b}(f):=\sup \sum_{k=1}^{n}\left|f\left(x_{k}\right)-f\left(x_{k-1}\right)\right|,
$$

where the least upper bound is taken over all (finite) partitions (4.7) of the interval $[a, b]$ (see [18]).

Lemma 4.1. For any positive $N$, there is a positive constant $\Upsilon=\Upsilon(N)$ not depending on $b$ such that

$$
\bigvee_{-N}^{N}\left\{\varrho_{b}(\lambda)\right\}=\sum_{-N \leq \lambda_{m, b}<N} \frac{1}{\alpha_{m, b}^{2}}=\varrho_{b}(N)-\varrho_{b}(-N)<\Upsilon
$$

Proof. Let $\sin \beta \neq 0$. Since $\varphi_{1}^{(1)}(x, \lambda)$ is continuous on the region

$$
\{(x, \lambda):-N \leq \lambda \leq N, a \leq x \leq c\}
$$


by condition $\varphi_{1}^{(1)}(a, \lambda)=\sin \beta$, there is a positive number $k$ and near by $a$ such that

$$
\frac{1}{k^{2}}\left(\int_{a}^{k} \varphi_{1}^{(1)}(x, \lambda) d x\right)^{2}>\frac{1}{2} \sin ^{2} \beta
$$

Let us define $f_{k}(x)=\left(\begin{array}{l}f_{k 1}(x) \\ f_{k 2}(x)\end{array}\right)$ by

$$
f_{k 1}(x)=0, f_{k 2}(x)=\left\{\begin{array}{cc}
\frac{1}{k}, & a \leq x<k \\
0, & x \geq k
\end{array}\right.
$$

From (4.6), (4.8) and (4.9), we get

$$
\begin{aligned}
\int_{a}^{k}\left(f_{k 1}^{2}(x)+f_{k 2}^{2}(x)\right) d x & =\frac{k-a}{k^{2}}=\int_{-\infty}^{\infty}\left(\frac{1}{k} \int_{a}^{k} \phi_{1}^{(1)}(x, \lambda) d x\right) d \varrho_{b}(\lambda) \\
& \geq \int_{-N}^{N}\left(\frac{1}{k} \int_{a}^{k} \phi_{1}^{(1)}(x, \lambda) d x\right)^{2} d \varrho_{b}(\lambda) \\
& >\frac{1}{2} \sin ^{2} \beta\left\{\varrho_{b}(N)-\varrho_{b}(-N)\right\},
\end{aligned}
$$

which proves the inequality (4.8). formula

If $\sin \beta=0$, then we define the function $f_{k}(x)=\left(\begin{array}{l}f_{k 1}(x) \\ f_{k 2}(x)\end{array}\right)$ by the

$$
f_{k 1}(x)=\left\{\begin{array}{cc}
\frac{1}{k^{2}}, & a \leq x<k \\
0, & x \geq k
\end{array} \quad, \quad f_{k 2}(x)=0\right.
$$

So, we obtain the inequality (4.8) by applying the Parseval equality.

Now, we will obtain an expansion into a Fourier series of resolvent if one knows the expansion of the function $f($.$) .$ 
By integration by parts, we find

$$
\begin{aligned}
\int_{a}^{c} & \left(\tau\left(y^{(1)}\right), \varphi_{m, b}^{(1)}(x)\right)_{E} d x+\delta \int_{c}^{b}\left(\tau\left(y^{(2)}\right), \varphi_{m, b}^{(2)}(x)\right)_{E} d x \\
= & \int_{a}^{c}\left[-y_{2}^{(1) \prime}(x)+p(x) y_{1}^{(1)}\right] \varphi_{m, b 1}^{(1)}(x) d x \\
& +\delta \int_{c}^{b}\left[-y_{2}^{(2) \prime}(x)+p(x) y_{1}^{(2)}\right] \varphi_{m, b 1}^{(2)}(x) d x \\
& +\int_{a}^{c}\left[y_{1}^{(1) \prime}(x)+r(x) y_{2}^{(1)}\right] \varphi_{m, b 2}^{(1)}(x) d x \\
& +\delta \int_{c}^{b}\left[y_{1}^{(2) \prime}(x)+r(x) y_{2}^{(2)}\right] \varphi_{m, b 2}^{(2)}(x) d x \\
= & \int_{a}^{c}\left[-\varphi_{m, b 2}^{(1) \prime}(x)+p(x) \varphi_{m, b 1}^{(1)}\right] y_{2}^{(1)} d x \\
& +\delta \int_{c}^{b}\left[-\varphi_{m, b 2}^{(2) \prime}(x)+p(x) \varphi_{m, b 1}^{(2)}\right] y_{2}^{(2)} d x \\
& +\int_{a}^{c}\left[\varphi_{m, b 1}^{(1) \prime}(x)+r(x) \varphi_{m, b 2}^{(1)}\right] y_{1}^{(1)} d x \\
& +\delta \int_{c}^{b}\left[-\varphi_{m, b 1}^{(2) \prime}(x)+p(x) \varphi_{m, b 1}^{(2)}\right] y_{1}^{(2)} d x \\
= & \lambda_{m, b} \int_{a}^{c}\left(y^{(1)}(x, \lambda), \varphi_{m, b}^{(1)}(x)\right)_{E} d x \\
& +\lambda_{m, b} \delta \int_{c}^{b}\left(y^{(2)}(x, \lambda) \varphi_{m, b}^{(2)}(x)\right)_{E} d x \\
= & \lambda{ }_{m, b} \gamma_{m}(\lambda)(m \in \mathbb{Z}),
\end{aligned}
$$

where

$$
\gamma_{m}(\lambda)=\int_{a}^{c}\left(y^{(1)}(x, \lambda), \varphi_{m, b}^{(1)}(x)\right)_{E} d x+\delta \int_{c}^{b}\left(y^{(2)}(x, \lambda) \varphi_{m, b}^{(2)}(x)\right)_{E} d x(m \in \mathbb{Z})
$$

Set

$$
\begin{gathered}
y(x, \lambda)=\sum_{m=-\infty}^{\infty} \gamma_{m}(\lambda) \varphi_{m, b}(x), \\
c_{m}=\int_{a}^{c}\left(f^{(1)}(x), \varphi_{m, b}^{(1)}(x)\right)_{E} d x+\delta \int_{c}^{b}\left(f^{(2)}(x), \varphi_{m, b}^{(2)}(x)\right)_{E} d x(m \in \mathbb{Z}) .
\end{gathered}
$$


Since $y(x, \lambda)$ satisfies the system $\tau(y)=\lambda y+f(x \in \Omega, f \in H)$ and conditions (2.2)-(2.4), we get

$$
\begin{aligned}
c_{m}= & \int_{a}^{c}\left(f^{(1)}(x), \varphi_{m, b}^{(1)}(x)\right)_{E} d x+\delta \int_{c}^{b}\left(f^{(2)}(x), \varphi_{m, b}^{(2)}(x)\right)_{E} d x \\
= & \int_{a}^{c}\left(\tau\left(y^{(1)}\right)(x), \varphi_{m, b}^{(1)}(x)\right)_{E} d x+\delta \int_{c}^{b}\left(\tau\left(y^{(2)}(x)\right), \varphi_{m, b}^{(2)}(x)\right)_{E} d x \\
& -\lambda \int_{a}^{c}\left(y^{(1)}(x), \varphi_{m, b}^{(1)}(x)\right)_{E} d x-\lambda \delta \int_{c}^{b}\left(y^{(2)}(x), \varphi_{m, b}^{(2)}(x)\right)_{E} d x \\
= & \left(\lambda_{m, b}-\lambda\right) \gamma_{m}(\lambda)(m \in \mathbb{Z}) .
\end{aligned}
$$

Then, we obtain

$$
\gamma_{m}(\lambda)=\frac{c_{m}}{\lambda_{m, b}-\lambda},(m \in \mathbb{Z})
$$

and

$$
y(x, \lambda)=\int_{a}^{b} G_{b}(x, t, \lambda) f(t) d t=\sum_{m=-\infty}^{\infty} \frac{c_{m}}{\lambda_{m, b}-\lambda} \varphi_{m, b}(x) .
$$

Hence, the expansion of the resolvent is

$$
\begin{aligned}
\left(R_{b} f\right)(x, z) & =\sum_{m=-\infty}^{\infty} \frac{\varphi_{m, b}(x)\left\langle f(.), \varphi_{m, b}(.)\right\rangle_{H}}{\alpha_{m, b}^{2}\left(\lambda_{m, b}-z\right)} \\
& =\int_{-\infty}^{\infty} \frac{\varphi(x, \lambda)}{\lambda-z}\langle f(.), \varphi(.)\rangle_{H} d \varrho_{b}(\lambda) .
\end{aligned}
$$

LEMma 4.2. Let $z$ be a non real number and $x$ be a fixed number. Then we have

$$
\int_{-\infty}^{\infty}\left\|\frac{\varphi(x, \lambda)}{\lambda-z}\right\|_{E}^{2} d \varrho_{b}(\lambda)<K
$$

Proof. Putting $f(x)=\varphi_{m, b}(x)$ in (4.10), we get

$$
\frac{1}{\alpha_{m, b}} \int_{a}^{b} G_{b}(x, t, z) \varphi_{m, b}(t) d t=\frac{\varphi_{m, b}(x)}{\alpha_{m, b}\left(\lambda_{m, b}-z\right)},
$$

since the eigenfunctions $\varphi_{m, b}(x)$ are orthogonal. Using (4.13), if we apply the Parseval equality to $G_{b}(x, t, z)$, we have

$$
\begin{aligned}
\int_{a}^{b}\left\|G_{b}(x, t, z)\right\|_{E}^{2} d x & =\sum_{m=-\infty}^{\infty} \frac{\left\|\varphi_{m, b}(x)\right\|_{E}^{2}}{\alpha_{m, b}^{2}\left|\lambda_{m, b}-z\right|^{2}} \\
& =\int_{-\infty}^{\infty}\left\|\frac{\varphi(x, \lambda)}{\lambda-z}\right\|_{E}^{2} d \varrho_{b}(\lambda) .
\end{aligned}
$$

Since the last integral convergent, the statement of lemma follows. 


\section{INTEGRAL REPRESENTATIONS FOR THE RESOLVENT OPERATOR IN SINGULAR CASE}

In this section, we will obtain integral representations for the resolvent in singular case.

Now, we recall that the following well-known theorems of Helly's.

THEOREM 5.1 ([18]). Let $\left(w_{n}\right)_{n \in \mathbb{N}}$ be a uniformly bounded sequence of real nondecreasing function on a finite interval $a \leq \lambda \leq b$. Then there exists a subsequence $\left(w_{n_{k}}\right)_{k \in \mathbb{N}}$ and a nondecreasing function $w$ such that

$$
\lim _{k \rightarrow \infty} w_{n_{k}}(\lambda)=w(\lambda), a \leq \lambda \leq b .
$$

TheOREM $5.2([18])$. Assume $\left(w_{n}\right)_{n \in \mathbb{N}}$ is a real, uniformly bounded, sequence of nondecreasing function on a finite interval $a \leq \lambda \leq b$, and suppose

$$
\lim _{n \rightarrow \infty} w_{n}(\lambda)=w(\lambda), a \leq \lambda \leq b .
$$

If $f$ is any continuous function on $a \leq \lambda \leq b$, then

$$
\lim _{n \rightarrow \infty} \int_{a}^{b} f(\lambda) d w_{n}(\lambda)=\int_{a}^{b} f(\lambda) d w(\lambda) .
$$

By Lemma 3, the set $\left\{\varrho_{b}(\lambda)\right\}$ is bounded. Using Theorems 5 and 6 , we can find a sequence $\left\{b_{k}\right\}$ such that the function $\varrho_{b_{k}}(\lambda)$ converge to a monotone function $\varrho(\lambda)$. Then we have a lemma.

LEMMA 5.3. Let $z$ be a non real number, and let $\varphi(x, \lambda)$ be as in (2.6). Then we have

$$
\int_{-\infty}^{\infty}\left\|\frac{\varphi(x, \lambda)}{\lambda-z}\right\|_{E}^{2} d \varrho(\lambda) \leq K,
$$

where $x$ be a fixed number.

ProOF. By the inequality (4.12), for arbitrary $\eta>0$, we have

$$
\int_{-\eta}^{\eta}\left\|\frac{\varphi(x, \lambda)}{\lambda-z}\right\|_{E}^{2} d \varrho_{b}(\lambda)<K .
$$

Letting $\eta \rightarrow \infty$ and $b \rightarrow \infty$, we get the desired result.

LEMma 5.4. For arbitrary $\eta>0$, we have the following inequalities.

$$
\int_{-\infty}^{-\eta} \frac{d \varrho(\lambda)}{\lambda^{2}}<\infty, \int_{\eta}^{\infty} \frac{d \varrho(\lambda)}{\lambda^{2}}<\infty .
$$

Proof. Since $\left\|\varphi_{m, b}(a, \lambda)\right\|_{E}^{2} \neq 0$, putting $x=a$ in (5.1), we get

$$
\int_{-\infty}^{\infty} \frac{d \varrho(\lambda)}{|\lambda-z|^{2}}<\infty
$$

and the statement of lemma follows. 
Lemma 5.5. Let $f(.) \in \mathcal{H}$, and let

$$
(R f)(x, z)=\int_{a}^{\infty} G(x, t, z) f(t) d t
$$

where

$$
G(x, t, z)= \begin{cases}\chi(x, z) \varphi^{T}(t, z), & t \leq x, x \neq c, t \neq c \\ \varphi(x, z) \chi^{T}(t, z), & t>x, x \neq c, t \neq c\end{cases}
$$

Then

$$
\begin{aligned}
& \int_{a}^{c}\|(R f)(x, z)\|_{E}^{2} d x+\delta \int_{c}^{\infty}\|(R f)(x, z)\|_{E}^{2} d x \\
& \leq \frac{1}{v^{2}}\left(\int_{a}^{c}\|f(x)\|_{E}^{2} d x+\delta \int_{c}^{\infty}\|f(x)\|_{E}^{2} d x\right), z=u+i v .
\end{aligned}
$$

Proof. For each $b>c$, it follows from (4.10) and the Parseval equality that

$$
\begin{aligned}
& \int_{a}^{c}\left\|\left(R_{b} f\right)(x, z)\right\|_{E}^{2} d x+\delta \int_{c}^{b}\left\|\left(R_{b} f\right)(x, z)\right\|_{E}^{2} d x \\
& =\sum_{m=-\infty}^{\infty} \frac{1}{\alpha_{m, b}^{2}\left|\lambda_{m, b}-z\right|^{2}}\left\{\left\langle f(.), \varphi_{m, b}(.)\right\rangle_{H}\right\}^{2} \\
& \leq \frac{1}{v^{2}} \sum_{m=-\infty}^{\infty} \frac{1}{\alpha_{m, b}^{2}}\left\{\left\langle f(.), \varphi_{m, b}(.)\right\rangle_{H}\right\}^{2} \\
& =\frac{1}{v^{2}}\left(\int_{a}^{c}\|f(x)\|_{E}^{2} d x+\delta \int_{c}^{b}\|f(x)\|_{E}^{2} d x\right) .
\end{aligned}
$$

Letting $b \rightarrow \infty$, we get the desired result.

The main result of the paper is the following theorem.

TheOREM 5.6. For every nonreal $z$ and for each $f(.) \in \mathcal{H}$, one has the following equality

$$
(R f)(x, z)=\int_{-\infty}^{\infty} \frac{\varphi(x, \lambda)}{\lambda-z} F(\lambda) d \varrho(\lambda),
$$

where

$$
F(\lambda)=\int_{a}^{c}\left(f(x), \varphi^{(1)}(x, \lambda)\right)_{E} d x+\delta \lim _{\xi \rightarrow \infty} \int_{c}^{\xi}\left(f(x), \varphi^{(2)}(x, \lambda)\right)_{E} d x,
$$

and $\varphi(x, \lambda)$ is defined by (2.6). 
PROOF. Let $\varsigma$ be an arbitrary positive number and the real-valued function

$$
\begin{aligned}
f_{\xi}(x) & =\left(\begin{array}{l}
f_{\xi 1}(x) \\
f_{\xi 2}(x)
\end{array}\right), f_{\xi 1}(x)=\left\{\begin{array}{ll}
f_{\xi 1}^{(1)}(x), & x \in[a, c) \\
f_{\xi 1}^{(2)}(x), & x \in(c, \xi]
\end{array},\right. \\
f_{\xi 2}(x) & = \begin{cases}f_{\xi 2}^{(1)}(x), & x \in[a, c) \\
f_{\xi 2}^{(2)}(x), & x \in(c, \xi]\end{cases}
\end{aligned}
$$

satisfies the following conditions:

1) $f_{\xi}(x)$ vanishes outside the set $[a, c) \cup(c, \xi], \xi>b$.

2) The function $f_{\xi}(x)$ has a continuous derivative.

3) $f_{\xi}(x)$ satisfy the conditions $(2.2)-(2.4)$.

Set

$$
F_{\xi}(\lambda)=\int_{a}^{c}\left(f_{\xi}^{(1)}(x), \varphi^{(1)}(x, \lambda)\right)_{E} d x+\delta \int_{c}^{\xi}\left(f_{\xi}^{(2)}(x), \varphi^{(2)}(x, \lambda)\right)_{E} d x
$$

From (4.11), we get

$$
\begin{aligned}
& \left(R_{b} f_{\xi}\right)(x, z) \\
& =\int_{-\infty}^{\infty} \frac{\varphi(x, \lambda)}{\lambda-z} F_{\xi}(\lambda) d \varrho_{b}(\lambda)=\int_{-\infty}^{-\varsigma} \frac{\varphi(x, \lambda)}{\lambda-z} F_{\xi}(\lambda) d \varrho_{b}(\lambda) \\
& \quad+\int_{-\varsigma}^{\varsigma} \frac{\varphi(x, \lambda)}{\lambda-z} F_{\xi}(\lambda) d \varrho_{b}(\lambda)+\int_{\varsigma}^{\infty} \frac{\varphi(x, \lambda)}{\lambda-z} F_{\xi}(\lambda) d \varrho_{b}(\lambda) \\
& =I_{1}+I_{2}+I_{3} .
\end{aligned}
$$

Now, we will estimate $I_{1}$. By (4.10), we get

$$
\begin{aligned}
\left|I_{1}\right| & =\left|\int_{-\infty}^{-\varsigma} \frac{\varphi(x, \lambda)}{\lambda-z} F_{\xi}(\lambda) d \varrho_{b}(\lambda)\right| \\
& =\left|\sum_{\lambda_{k, b}<-\varsigma} \frac{\varphi_{k, b}(x)}{\alpha_{k, b}^{2}\left(\lambda_{k, b}-z\right)}\left\{\begin{array}{c}
\int_{a}^{c}\left(f_{\xi}^{(1)}(x), \varphi_{k, b}^{(1)}(x)\right)_{E} d x \\
+\delta \int_{c}^{\xi}\left(f_{\xi}^{(2)}(x), \varphi_{k, b}^{(2)}(x)\right)_{E} d x
\end{array}\right\}\right| \\
& \leq\left(\sum_{\lambda_{k, b}<-\varsigma} \frac{\left\|\varphi_{k, b}(x)\right\|_{E}^{2}}{\alpha_{k, b}^{2}\left|\lambda_{k, b}-z\right|^{2}}\right)^{1 / 2} \\
& \times\left(\sum_{\lambda_{k, b}<-\varsigma} \frac{1}{\alpha_{k, b}^{2}}\left[\begin{array}{c}
+\delta \int_{c}^{c}\left(f_{\xi}^{(1)}(x), \varphi_{k, b}^{(1)}(x)\right)_{E} d x \\
\left.\int_{\xi}^{(2)}(x), \varphi_{k, b}^{(2)}(x)\right)_{E} d x
\end{array}\right)^{1 / 2}\right.
\end{aligned}
$$


By integration by parts, we find

$$
\begin{aligned}
& \int_{a}^{c}\left(f_{\xi}^{(1)}(x), \varphi_{k, b}^{(1)}(x, \lambda)\right)_{E} d x+\delta \int_{c}^{\xi}\left(f_{\xi}^{(2)}(x), \varphi_{k, b}^{(2)}(x, \lambda)\right)_{E} d x \\
& =\frac{1}{\lambda_{k, b}}\left\{\begin{array}{c}
\int_{a}^{c} f_{\xi 1}^{(1)}(x)\left\{-\varphi_{k, b 2}^{(1) \prime}(x)+p(x) \varphi_{k, b 1}^{(1)}(x)\right\} d x \\
+\delta \int_{c}^{\xi} f_{\xi 1}^{(2)}(x)\left\{-\varphi_{k, b 2}^{(2) \prime}(x)+p(x) \varphi_{k, b 1}^{(2)}(x)\right\} d x
\end{array}\right\} \\
& +\frac{1}{\lambda_{k, b}}\left\{\begin{array}{c}
\int_{a}^{c} f_{\xi 2}^{(1)}(x)\left\{\varphi_{k, b 1}^{(1) \prime}(x)+r(x) \varphi_{k, b 2}^{(1)}(x)\right\} d x \\
+\delta \int_{c}^{\xi} f_{\xi 2}^{(2)}(x)\left\{\varphi_{k, b 1}^{(2) \prime}(x)+r(x) \varphi_{k, b 2}^{(2)}(x)\right\} d x
\end{array}\right\} \\
& =\frac{1}{\lambda_{k, b}}\left\{\begin{array}{c}
\int_{a}^{c} \varphi_{k, b 1}^{(1)}(x)\left\{-f_{\xi 2}^{(1) \prime}(x)+p(x) f_{\xi 1}^{(1)}(x)\right\} d x \\
+\delta \int_{c}^{\xi} \varphi_{k, b 1}^{(2)}(x)\left\{-f_{\xi 2}^{(2) \prime}(x)+p(x) f_{\xi 1}^{(2)}(x)\right\} d x
\end{array}\right\} \\
& +\frac{1}{\lambda_{k, b}}\left\{\begin{array}{c}
\int_{a}^{c} \varphi_{k, b 2}^{(1)}(x)\left\{f_{\xi 1}^{(1) \prime}(x)+r(x) f_{\xi 2}^{(1)}(x)\right\} d x \\
+\delta \int_{c}^{\xi} \varphi_{k, b 2}^{(2)}(x)\left\{f_{\xi 1}^{(2) \prime}(x)+r(x) f_{\xi 2}^{(2)}(x)\right\} d x
\end{array}\right\} .
\end{aligned}
$$

By Lemma 4, we have

$$
\left|I_{1}\right| \leq \frac{K^{1 / 2}}{\varsigma^{2}}\left(\sum_{\lambda_{k, b}<-\varsigma} \frac{1}{\alpha_{k, b}^{2}}\left[\begin{array}{c}
\int_{a}^{c}\left(h_{\xi}(x), \varphi_{k, b}(x)\right)_{E} d x \\
+\delta \int_{c}^{\xi}\left(h_{\xi}(x), \varphi_{k, b}(x)\right)_{E} d x
\end{array}\right]^{2}\right)^{1 / 2}
$$

where

$$
h_{\xi}(t)=\left(\begin{array}{c}
-f_{\xi 2}^{\prime}(x)+p(x) f_{\xi 1}(x) \\
f_{\xi 1}^{\prime}(x)+r(x) f_{\xi 2}(x)
\end{array}\right)
$$

Using Bessel inequality, we get

$$
\left|I_{1}\right| \leq \frac{K^{1 / 2}}{\varsigma}\left[\int_{a}^{c}\left\|h_{\xi}(x)\right\|_{E}^{2} d x+\delta \int_{c}^{\xi}\left\|h_{\xi}(x)\right\|_{E}^{2} d x\right]^{1 / 2}=\frac{C_{1}}{\varsigma} .
$$

By similar method, one can prove that $\left|I_{3}\right| \leq \frac{C_{2}}{\varsigma}$. Then $I_{1}$ and $I_{3}$ tend to zero as $\varsigma \rightarrow \infty$, uniformly in $b$. Using Theorems 5 and 6 in (5.4), we obtain

$$
\left(R f_{\xi}\right)(x, z)=\int_{-\infty}^{\infty} \frac{\varphi(x, \lambda)}{\lambda-z} F_{\xi}(\lambda) d \varrho(\lambda) .
$$

As is known, if $f(.) \in \mathcal{H}$, then one can find a sequence $\left\{f_{\xi}(x)\right\}_{\xi=1}^{\infty}$ which satisfy the previous conditions and tend to $f(x)$ as $\xi \rightarrow \infty$. From the Parseval equality, the sequence of Fourier transform converges to the transform of $f(x)$. By Lemmas 7 and 9, we can pass to the limit $\xi \rightarrow \infty$ in (5.7). So, we obtain the assertion of the theorem. 


\section{The formulas for the Titchmarsh-Weyl Function and the SPECTRAL FUNCTION}

In this section, we will derive formulas for the Titchmarsh-Weyl function $m(z)$ and the spectral function $\varrho(\lambda)$ with the help of the integral representation of the resolvent.

Firstly, we recall the Stieltjes inversion formula. Let $\sigma(\lambda)=\sigma_{1}(\lambda)+$ $i \sigma_{2}(\lambda)$ be a complex valued function of bounded variation on the entire line. We put

$$
\begin{aligned}
\varphi(z) & =\int_{-\infty}^{\infty} \frac{d \sigma(\lambda)}{z-\lambda}, \psi(\nu, \tau)=\frac{\operatorname{sgn} \tau}{\pi} \frac{\varphi(z)-\varphi(\bar{z})}{2 i} \\
& =-\frac{1}{\pi} \int_{-\infty}^{\infty} \frac{|\tau| d \sigma(\lambda)}{(\lambda-\nu)^{2}+\tau^{2}}, z=\nu+i \tau
\end{aligned}
$$

THEOREM 6.1 ([20]). If the points $a, b$ are points of continuity of $\sigma(\lambda)$, then we have

$$
\sigma(b)-\sigma(a)=-\lim _{\tau \rightarrow 0} \int_{a}^{b} \psi(\nu, \tau) d \nu
$$

THEOREM 6.2. (i) For any nonreal $z$, one has

$$
m(z)-m\left(z_{0}\right)=\int_{-\infty}^{\infty}\left[\frac{1}{\lambda-z}-\frac{1}{\lambda-z_{0}}\right] d \varrho(\lambda),\left(\operatorname{Im} z_{0} \neq 0\right) .
$$

(ii) If $\lambda$ and $\mu$ are points of continuity of $\varrho(\lambda)$, then one has

$$
\varrho(\lambda)-\varrho(\mu)=-\frac{1}{\pi} \lim _{\tau \rightarrow 0} \int_{\mu}^{\lambda} \operatorname{Im}\{m(\nu+i \tau)\} d \nu, z=\nu+i \tau, \tau>0 .
$$

Proof. (i) Since $f(x)$ is arbitrary, it follows from (5.3) that

$$
G(t, u, z)=\int_{-\infty}^{\infty} \frac{\varphi(t, \lambda) \varphi^{T}(u, \lambda)}{\lambda-z} d \varrho(\lambda) .
$$

Hence,

$$
G(t, u, z)-G\left(t, u, z_{0}\right)=\int_{-\infty}^{\infty} \varphi(t, \lambda) \varphi^{T}(u, \lambda)\left[\frac{1}{\lambda-z}-\frac{1}{\lambda-z_{0}}\right] d \varrho(\lambda) .
$$

Since there are matrices on both sides in (6.3), their corresponding elements are equal. So, using (4.1) and the definition of the product $\varphi(t, \lambda) \varphi^{T}(u, \lambda)$, and then putting $t=u=a$ and taking the initial conditions (2.7), we get

$$
\begin{aligned}
& \{\cos \beta+m(z) \sin \beta\} \sin \beta-\left\{\cos \beta+m\left(z_{0}\right) \sin \beta\right\} \sin \beta \\
& =\int_{-\infty}^{\infty} \sin ^{2} \beta\left[\frac{1}{\lambda-z}-\frac{1}{\lambda-z_{0}}\right] d \varrho(\lambda)
\end{aligned}
$$


i.e.,

$$
m(z)-m\left(z_{0}\right)=\int_{-\infty}^{\infty}\left[\frac{1}{\lambda-z}-\frac{1}{\lambda-z_{0}}\right] d \varrho(\lambda) .
$$

(ii) From (6.1), we get

$$
\begin{aligned}
\psi(\nu, \tau) & =\frac{\operatorname{sgn} \tau}{\pi} \frac{m(z)-m(\bar{z})}{2 i} \\
& =-\frac{1}{\pi} \int_{-\infty}^{\infty} \frac{|\tau| d \varrho(\lambda)}{(\lambda-\nu)^{2}+\tau^{2}} .
\end{aligned}
$$

By Theorem 11, we have

$$
\varrho(\lambda)-\varrho(\mu)=-\lim _{\tau \rightarrow 0} \int_{\mu}^{\lambda} \psi(\nu, \tau) d \nu .
$$

Since $m(\bar{z})=\overline{m(z)}$, it follows that

$$
\psi(\nu, \tau)=\frac{\operatorname{sgn} \tau}{\pi} \frac{m(z)-m(\bar{z})}{2 i}=\frac{\operatorname{sgn} \tau}{\pi} \operatorname{Im}\{m(z)\} .
$$

For $\tau>0$, we obtain the formula (6.2) using (6.4) and (6.5). Thus, the theorem is proved.

\section{REFERENCES}

[1] Z. Akdoğan, M. Demirci and O. Sh. Mukhtarov, Green function of discontinuous boundary value problem with transmission conditions, Math. Meth. Appl. Sci. 30 (2007), 1719-1738.

[2] B. P. Allahverdiev and E. Uğurlu, Scattering and spectral problems of the direct sum Sturm-Liouville operators, Appl. Comput. Math. 16 (2017), 257-268.

[3] B. P. Allahverdiev and E. Uğurlu, On dilation, scattering and spectral theory for twointerval singular differential operators, Bull. Math. Soc. Sci. Math. Roumanie 58(106) (2015), 383-392.

[4] B. P. Allahverdiev, E. Bairamov and E. Uğurlu, Eigenparameter dependent SturmLiouville problems in boundary conditions with transmission conditions, J. Math. Anal. Appl. 401 (2013), 388-396.

[5] B. P. Allahverdiev and E. Uğurlu, Spectral analysis of the direct sum Hamiltonian operators, Quaest. Math. 39 (2016), 733-750.

[6] B. P. Allahverdiev and H. Tuna, Titchmarsh-Weyl theory for Dirac systems with transmission conditions, Mediterr. J. Math. 15 (2018), no. 4, Art. 151.

[7] B. P. Allahverdiev and H. Tuna, Spectral expansion for singular Dirac system with impulsive conditions, Turkish J. Math. 42 (2018), 2527-2545.

[8] R. Kh. Amirov, On a system of Dirac differential equations with discontinuity conditions inside an interval, Ukrainian Math. J. 57 (2005), 712-727.

[9] E. Bairamov and E. Uğurlu, The determinants of dissipative Sturm-Liouville operators with transmission conditions, Math. Comput. Modelling 53 (2011), 805-813.

[10] I. Dehghani and A. J. Akbarfam, Resolvent operator and self-adjointness of SturmLiouville operators with a finite number of transmission conditions, Mediterr. J. Math. 11 (2014), 447-462.

[11] Ş. Faydaoğlu and G. Sh. Guseinov, Eigenfunction expansion for a Sturm-Liouville boundary value problem with impulse, Int. J. Pure Appl. Math. 8 (2003), 137-170. 
[12] Ş. Faydaoğlu and G. Sh. Guseinov, An expansion result for a Sturm-Liouville eigenvalue problem with impulse, Turkish J. Math. 34 (2010), 355-366.

[13] Y. Güldü, On discontinuous Dirac operator with eigenparameter dependent boundary and two transmission conditions, Bound. Value Probl. 2016, Paper No. 135.

[14] F. Hıra and N. Altınışı, Eigenvalue problem for discontinuous Dirac system with eigenparameter in a transmission condition, Gen. Math. Notes 31 (2015), 72-84.

[15] A. Kablan and T. Özden, A Dirac system with transmission condition and eigenparameter in boundary condition, Abstr. Appl. Anal. 2013, Art. ID 395457.

[16] B. Keskin and A. S. Ozkan, Inverse spectral problems for Dirac operator with eigenvalue dependent boundary and jump conditions, Acta Math. Hungarica 130 (2011), 309-320.

[17] K. Knopp, Elements of the Theory of Functions, Dover, New York, 1952.

[18] A. N. Kolmogorov and S. V. Fomin, Introductory Real Analysis, Translated by R. A. Silverman, Dover Publications, New York, 1970

[19] F. R. Lapwood and T. Usami, Free Oscillations of the Earth, Cambridge University Press, Cambridge, 1981.

[20] B. M. Levitan and I. S. Sargsjan, Sturm-Liouville and Dirac Operators. Mathematics and its Applications (Soviet Series), Kluwer Academic Publishers Group, Dordrecht, 1991.

[21] K. Li, J. Sun and X. Hao, Weyl function of Sturm-Liouville problems with transmission conditions at finite interior points, Mediter. J. Math. 14 (2017), no. 5, Art. 189.

[22] A. V. Likov and Yu. A. Mikhailov, The Theory of Heat and Mass Transfer, Translated from Russian by I. Shechtman, Israel Program for Scientific Translations, Jerusalem, 1965.

[23] O. N. Litvinenko and V. I. Soshnikov, The Theory of Heteregenous Lines and their Applications in Radio Engineering, Radio, Moscow (1964) (in Russian).

[24] R. Kh. Mamedov and O. Akcay, Inverse eigenvalue problem for a class of Dirac operators with discontinuous coefficient, Bound. Value Probl. 2014, 2014:110.

[25] O. Sh. Mukhtarov and M. Kadakal, Some spectral properties of one Sturm-Liouville type problem with discontinuous weight, Siberian Math. J. 46 (2005), 681-694.

[26] O. Sh. Mukhtarov, Discontinuous boundary-value problem with spectral parameter in boundary conditions, Turkish J. Math. 18 (1994), 183-192.

[27] O. Sh. Mukhtarov and E. Tunç, Eigenvalue problems for Sturm Liouville equations with transmission conditions, Israel J. Math. 144 (2004), 367-380.

[28] O. Sh. Mukhtarov and S. Yakubov, Problems for differential equations with transmission conditions, Applicable Anal. 81 (2002), 1033-1064.

[29] B. Thaller, The Dirac Equation, Springer, 1992.

[30] M. M. Tharwat and A. H. Bhrawy, Computation of eigenvalues of discontinuous Dirac system using Hermite interpolation technique, Adv. Difference Equ. 2012, 2012:59.

[31] E. C. Titchmarsh, Eigenfunction Expansions Associated with Second-Order Differential Equations, Part I. Second Edition Clarendon Press, Oxford, 1962.

[32] H. Tuna and A. Eryllmaz, Dissipative Sturm-Liouville operators with transmission conditions, Abstr. Appl. Anal. 2013, Art. ID 248740.

[33] J. Weidmann, Spectral Theory of Ordinary Differential Operators, Lecture Notes in Mathematics, 1258, Springer, Berlin, 1987.

[34] A. Wang, J. Sun, X. Hao and S. Yao, Completeness of eigenfunctions of SturmLiouville problems with transmission conditions, Meth. Appl. Anal. 16 (2009), 299312.

[35] A. Wang and A. Zettl, Eigenvalues of Sturm-Liouville problems with discontinuous boundary conditions, Elect. J. Differ. Equ. 2017, Paper No. 127. 
[36] C. F. Yang and G. L. Yuan, Determination of Dirac operator with eigenvaluedependent boundary and jump conditions, Appl. Anal. 94 (2015), 1460-1478.

[37] A. Zettl, Adjoint and self-adjoint boundary value problems with interface conditions, SIAM J. Appl. Math. 16 (1968), 851-859.

\section{Rezolventni operator singularnog Diracovog sustava s uvjetima transmisije}

\section{Bilender P. Allahverdiev i Hüseyin Tuna}

SAŽETAK. U ovom članku se proučava rezolventni operator jednodimenzionalnog singularnog Diracovog operatora s uvjetima transmisije. Proučava se Titchmarsh-Weylova funkcija ovog problema. Također se konstruiraju Greenova funkcija i spektralna funkcija za regularni i singularni problem. Pomoću tih funkcija dobiva se razvoj u Fourierov red rezolvente u regularnom slučaju. Nadalje, dajemo integralnu reprezentaciju u terminima spektralne funkcije za rezolventu ovog operatora s uvjetima transmisije u singularnom slučaju. Naposljetku, dobivamo formulu za Titchmarsh-Weylovu funkciju u terminima spektralne funkcije singularnog Diracovog sustava.

Bilender P. Allahverdiev

Department of Mathematics

Süleyman Demirel University,

32260 Isparta, Turkey

E-mail: bilenderpasaoglu@sdu.edu.tr

Hüseyin Tuna

Department of Mathematics

Mehmet Akif Ersoy University,

15030 Burdur, Turkey

E-mail: hustuna@gmail.com

Received: 20.2 .2018 .

Revised: 22.6.2018.; 27.8.2018.

Accepted: 25.9.2018. 
